\title{
AMENDMENTS
}

\section{Author Correction: Human antibodies to the dengue virus E-dimer epitope have therapeutic activity against Zika virus infection}

Estefania Fernandez, Wanwisa Dejnirattisai, Bin Cao, Suzanne M Scheaffer, Piyada Supasa, Wiyada Wongwiwat, Prabagaran Esakky, Andrea Drury, Juthathip Mongkolsapaya, Kelle H Moley, Indira U Mysorekar, Gavin R Screaton and Michael S Diamond

Correction to: Nature Immunology https://www.nature.com/articles/ni.3849, published online 25 September 2017.

In the version of this article originally published, the label ('Infection (\%)') along the vertical axis of each plot in Fig. 1d was incorrect. The correct label is 'Neutralization (\%)'.

Published online: 30 January 2020

https://doi.org/10.1038/s41590-019-0560-5

(๑) The Author(s), under exclusive licence to Springer Nature America, Inc. 2019

\section{Author Correction: The neuropeptide VIP confers anticipatory mucosal immunity by regulating ILC3 activity}

Cyril Seillet (D), Kylie Luong (D), Julie Tellier, Nicolas Jacquelot, Rui Dong Shen, Peter Hickey (D), Verena C. Wimmer, Lachlan Whitehead, Kelly Rogers, Gordon K. Smyth (D), Alexandra L. Garnham, Matthew E. Ritchie (D) and

Gabrielle T. Belz iD

Correction to: Nature Immunology https://doi.org/10.1038/s41590-019-0567-y, published online 23 December 2019.

In the version of this article initially published, affiliation 4 was shown as "Diamantina Institute, University of Queensland, Brisbane, Queensland, Australia”. The correct affiliation is "The University of Queensland Diamantina Institute, The University of Queensland, Brisbane, Queensland, Australia." In Fig. 5d, the labels for the two panels were switched; the left panel should be labeled "IL-7 + IL-23" and the right panel should be labeled "IL-7." The units for the concentrations of dibutyryl in this panel were reported as "nM"; however, they should be " $\mu$ M." In Fig. 5e, the concentrations of VIP and BAY 55-9837 were reported as $100 \mathrm{mM}$ in the figure and the caption; however, the concentrations of both were $100 \mathrm{nM}$. In Fig. 5f, the concentration of VIP was reported as $100 \mathrm{mM}$; however, the concentra-

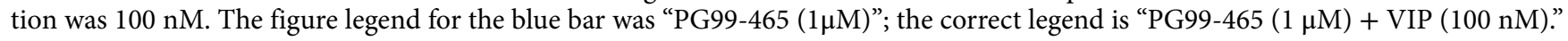
In Extended Data Fig. 4, the figure title read "Frequency of lymphocytes in the small intestine of food-restricted mice." The correct title is "Total number of lymphocytes in the small intestine of mixed bone-marrow chimeric mice." The figure caption stated "Cells derived from wild-type $\left(\mathrm{CD} 45.1^{+} \mathrm{CD} 45.2^{+}\right)$bone marrow are indicated in red and cells from Arntl ${ }^{\mathrm{AL}-7 \mathrm{R}}\left(\mathrm{CD} 45.2^{+/+}\right)$are shown in blue." The caption should say "Cells derived from wild-type (CD45. $1^{+}$CD $45.2^{+}$) bone marrow are indicated in white and cells from Arntl $^{\mathrm{AL}-7 \mathrm{R}}\left(\mathrm{CD} 45.2^{+/+}\right)$are shown in black." The errors have been corrected in the HTML and PDF versions of the article.

Published online: 30 January 2020

https://doi.org/10.1038/s41590-020-0606-8

๑ The Author(s), under exclusive licence to Springer Nature America, Inc. 2020 